\title{
Alternating Direction Implicit Methods for FDTD Using the Dey-Mittra Embedded Boundary Method
}

\author{
T.M. Austin*, J.R. Cary, D.N. Smithe and C. Nieter
}

Tech-X Corporation 5621 Arapahoe Ave. Suite A Boulder, CO 80303, USA

\begin{abstract}
The alternating direction implicit (ADI) method is an attractive option to use in avoiding the CourantFriedrichs-Lewy (CFL) condition that limits the size of the time step required by explicit finite-difference time-domain (FDTD) methods for stability. Implicit methods like Crank-Nicholson offer the same advantages as ADI methods but they do not rely on simple, one-dimensional, tridiagonal system solves for which there are well-known fast solution methods. To date, the ADI method applied to the FDTD method for curved domains has been used within the context of subgridding (i.e., local refinement) or for stairstepped boundaries that are only first-order accurate. A popular secondorder accurate approach to representing smooth domains with the FDTD method is the Dey-Mittra embedded boundary method. However, to be useful in a realistic setting, the cells with only a small fraction of their volume inside the domain need to be discarded from simulations for stability considerations or else the time step size will be prohibitively small. Using the ADI method instead of the explicit method implies that time step can be chosen to depend on accuracy and no cells need discarding. We show in this paper the ability to maintain stability beyond the CFL limit for the Dey-Mittra method without discarding any cells. We also consider convergence of the ADI method as compared to the standard explicit method that is limited by the CFL condition.
\end{abstract}

\section{INTRODUCTION}

The alternating direction implicit (ADI) method is a powerful implicit method for solving a finite-difference time-domain (FDTD) discretization of Maxwell's equations. This method (ADI-FDTD) consists of a series of simple, one-dimensional, tridiagonal system solves in contrast to a single large system solve as is required by the CrankNicholson method. The majority of the work on the ADIFDTD method has focused on simple, rectangular domains (cf. [1-4]) and avoided modeling curved domains like those found in complex accelerator structures.

One approach to modeling curved domains is to use a subgridding scheme on the boundary [5]. For such a subgridding scheme, a hybrid ADI-FDTD method was proposed in Ref. [6] that uses an ADI method on the higher resolved Yee cells and an explicit method on the coarser cells. In this work, instead of using subgridding, we address the boundary with schemes that alter the discrete equations in the cells cut by the boundaries, i.e., the cut-cells.

The original approach proposed by Yee in Ref. [7] for addressing the cut-cells was the stairstepping approach. In stairstepping, a cut-cell is labelled either interior or exterior depending on the location of the cut-cell center. See Fig. (1a). A more recent and accurate approach was proposed by Dey and Mittra in Ref. [8]. The Dey-Mittra approach accurately includes the fractional face areas and edge lengths of the cut-cells in the explicit method with the condition that small fractional face areas can cause a significant reduction in the time step to maintain stability. In this paper, we consider

\footnotetext{
*Address correspondence to this author at the Tech-X Corporation 5621 Arapahoe Ave. Suite A Boulder, CO 80303, USA;

E-mail: austin@txcorp.com
}

the FDTD method using a Dey-Mittra approach for the cutcells, which we refer to as the EXP-FDTD approach.

As described in Ref. [8], cut-cells with restrictively small fractional areas can be discarded from the simulation to maintain a reasonable time step. If too many cells are discarded, then the method can become first-order. Thus, a method is needed that employs the Dey-Mittra second-order approach but does not lead to restrictively small time steps and the reduction in convergence order. For this reason, we propose the ADI-FDTD method to be used in combination with the Dey-Mittra embedded boundary method. We will show that the ADI-FDTD method is stable at any time step and yields accurate frequency calculations for the 2D Palevsky-Bekefi A6 magnetron [9].

The outline of the paper is as follows. In the next section, we introduce the Dey-Mittra embedded boundary method that is used to model curved domains with the FDTD method and then we introduce the ADI-FDTD algorithm. We then briefly introduce the frequency extraction method of Ref. [10] and present results for the 2D A6 magnetron that is modeled using the FDTD method combined with the DeyMittra boundary algorithm. We consider a frequency extraction algorithm that requires time domain simulations using either the EXP-FDTD method or the ADI-FDTD method. We explore the accuracy of the various approaches and their stability. We end with a discussion of these results and a conclusion.

\section{METHODOLOGY}

\subsection{Dey-Mittra Embedded Boundary Method}

The classical FDTD method is the Yee method [7] used for solving Maxwell's equations on a lattice grid. In three dimensions, electric field values are located at the centers of 
(a) Stairstepping

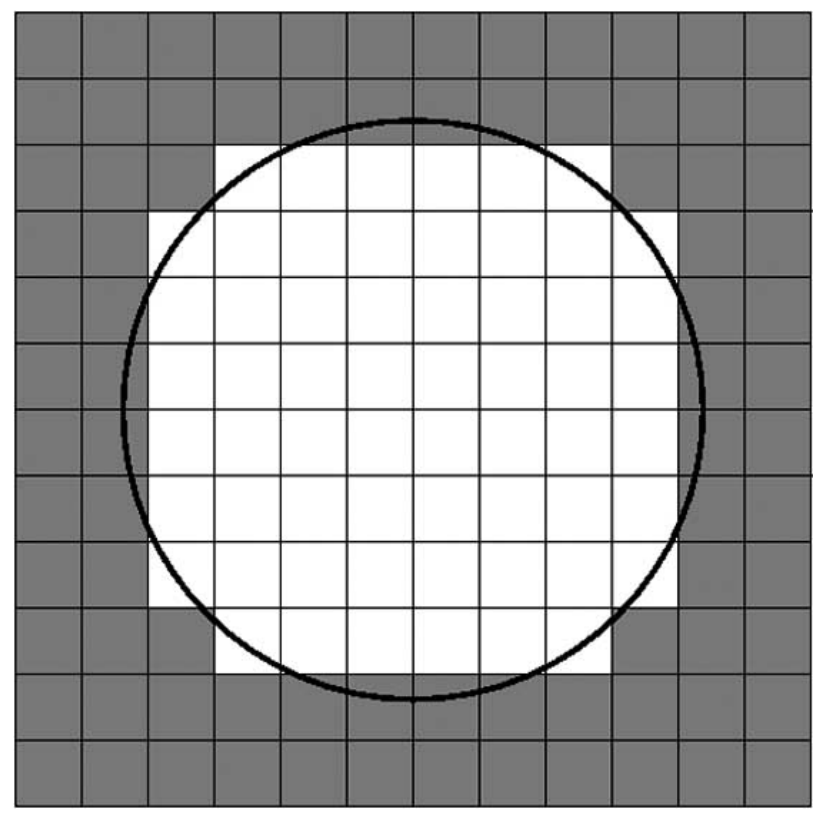

(b) Dey-Mittra

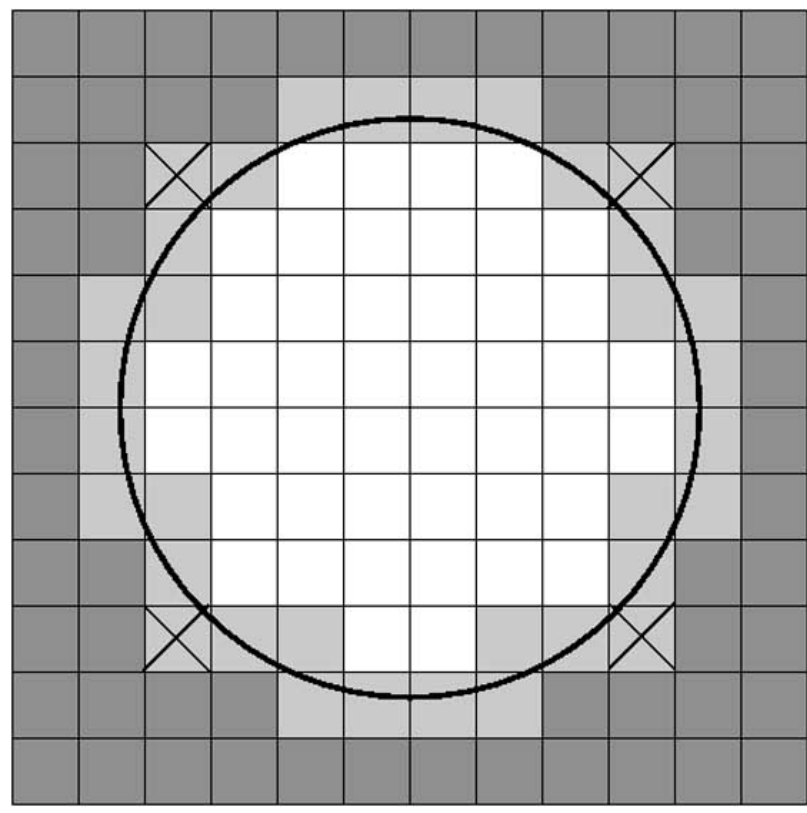

Fig. (1). (a) Approximating a curved boundary with a stairstepping approach, where the standard Yee algorithm is performed on the white cells, while the dark gray cells are in the metal boundary and left out of the computations. (b) Approximating with the DeyMittra embedded boundary method where the classical Yee method is used on all white cells, a Dey-Mittra embedded boundary method on the light gray cells, and the dark gray cells are in the metal boundary and left out of the computations. The X'd cells have only a fractional area in the domain and are assigned to be a part of the metal boundary.

the edges of a cubic cell and magnetic field values are located at the centers of the faces of a cubic cell. The Yee method is second-order accurate in time and space and preserves divergence-free quantities. The original approach for modeling embedded boundaries proposed by Yee in Ref. [7] stairsteps the boundary as in Fig. (1a). Because this is only a first-order method, the development of higher-order methods has been a high priority. In 1997 Dey and Mittra presented an embedded boundary algorithm that with some restrictions yields an overall second-order method [8]. The Dey-Mittra algorithm is the boundary approach used in the VORPAL computational framework [11], and thus has been extensively used and tested in Refs. [12, 13].

To describe the Dey-Mittra embedded boundary method, we consider the $x$-component of the equation for Faraday's Law in Maxwell's equation as $\partial B_{x} / \partial t=-(\nabla \times \mathbf{E})_{x}$. The finite difference version of this equation is given by

$$
\begin{aligned}
& B_{x ; i, j+1 / 2, k+1 / 2}^{n+1 / 2}=B_{x ; i, j+1 / 2, k+1 / 2}^{n-1 / 2}+\frac{\Delta t}{A_{y z}} \times \\
& \left(-E_{z ; i, j+1, k+1 / 2}^{n} \cdot l_{z}+E_{z ; i, j, k+1 / 2}^{n} \cdot l_{z}+E_{y ; i, j+1 / 2, k+1}^{n} \cdot l_{y}-E_{y ; i, j+1 / 2, k}^{n} \cdot l_{y}\right)
\end{aligned}
$$

where $B_{x, i, j+1 / 2, k+1 / 2}^{n+1 / 2}$ is the $z$-component of the magnetic field in cell $(i, j, k)$ at time $t=(n+1 / 2) \Delta t$. Additionally, $l_{y}$ and $l_{z}$ are the lengths of the cell edges on which $E_{y}$ and $E_{z}$ are located, and $A_{y z}$ is the cell area of the face centered at $B_{x}$. In Eq. (1) the electric field values are located on the edges of a face of a cell with the magnetic field value centered on the face.

The Dey-Mittra embedded boundary method redefines Eq. (1) when a metal boundary cuts through the cell face as in Fig. (2). A more accurate approach than stairstepping is needed to achieve a second-order method. The Dey-Mittra embedded boundary method sets the lengths ( $l_{y}$ and $\left.l_{z}\right)$ and the areas $\left(A_{y z}\right)$ in Eq. (1) to account for the cut-cell lengths and areas. See Fig. (2) for reference. The equivalent equation to Eq. (1) for the electric field update is advanced as with the Yee method but setting the electric field to zero when the corresponding edge is contained entirely within the metal boundary.

In Ref. [14], the authors describe the advantages and disadvantages of the Dey-Mittra embedded boundary algorithm. Of chief concern is the effect of the cut-cells on the maximum stable time step permitted. For the Yee algorithm, the time step for stability is limited by the CFL condition. As detailed in Ref. [14], the stability condition derived from the Gerschgorin Circle Theorem states that $\Delta t$ is related to $\Delta x, \Delta y$, and $\Delta z$ by

$$
\Delta t \leq \frac{c}{\sqrt{\frac{1}{\Delta x^{2}}+\frac{1}{\Delta y^{2}}+\frac{1}{\Delta z^{2}}}}
$$

where $c$ is the speed-of-light. As a result, the time step is determined by the stability of the numerical method, not the desired accuracy. The effect of a cut-cell on stability is to further restrict the time step because the cell size is being reduced when a cell is cut. Again, this is detailed in Ref. [14] where the authors introduce a factor, $f_{D M} \in[0,1]$, that acts as 
a threshold on the size of a cut-cell. For example, setting $f_{D M}=0.5$ ensures that a cell with a local Courant evaluation that is less than $50 \%$ of the nominal value is excluded from computations. This implies the time step, $\Delta t$, has the new stability condition given by

$$
\Delta t \leq 0.5 \cdot \Delta t_{C F L} \leq \frac{0.5 \cdot c}{\sqrt{\frac{1}{\Delta x^{2}}+\frac{1}{\Delta y^{2}}+\frac{1}{\Delta z^{2}}}} .
$$

Other disadvantages such as a reduction in order of convergence from second-order to first-order due to the deletion of too many cut-cells is discussed in Ref. [14]. In conclusion, an approach that uses the same set of equations but does not suffer from the effect of cut-cells on time step size and convergence is needed.

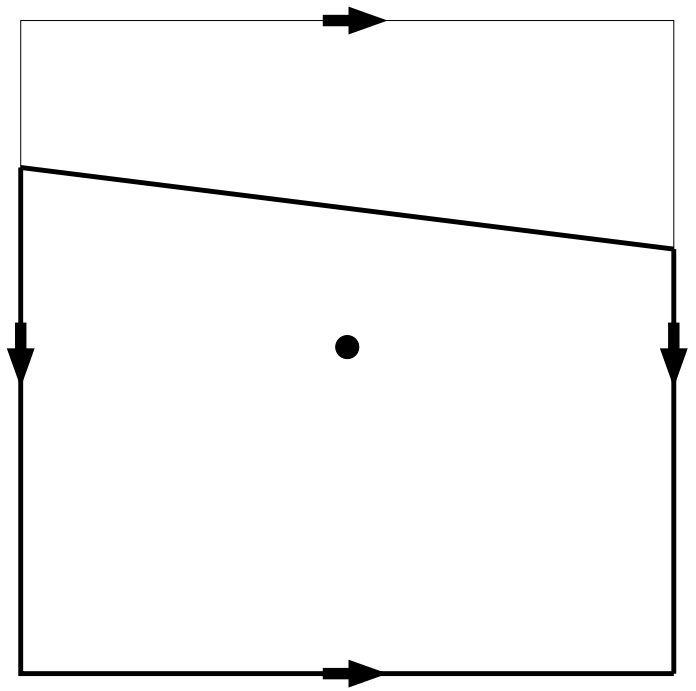

Fig. (2). A cut-cell in the Dey-Mittra embedded boundary algorithm is handled in the Faraday update (above) by adjusting the length $(l)$ and area values $(A)$ according to their fraction within the domain. The equivalent Ampere update for the electric field (not shown) is altered by setting the electric field value to zero for cells contained entirely within the metal boundary.

\subsection{Alternating Direction Implicit Method}

The alternating direction implicit method is an implicit method that solves a set of simple, one-dimensional, tridiagonal systems as part of the time step update. There is no CFL condition that limits the time step and thus no effect of fractional cut-cells from the Dey-Mittra method. Hence, it is an ideal candidate for use with the Dey-Mittra embedded boundary method. Furthermore, recently in Ref. [15], the authors presented a new version of the ADI-FDTD method. We briefly summarize the method here and then describe the translation to handling the embedded boundary equations from the Dey-Mittra approach.

To present the ADI-FDTD method, we express the Maxwell's equations as

$$
\begin{aligned}
& \frac{\partial \mathbf{B}}{\partial t}=-\nabla \times \mathbf{E} \\
& \frac{\partial \mathbf{E}}{\partial t}=c^{2} \nabla \times \mathbf{B}-\frac{\mathbf{J}}{\varepsilon_{0}},
\end{aligned}
$$

and note that these equations can be written as

$$
\frac{\partial \mathbf{W}}{\partial t}=\mathbf{S}+(\mathbf{P}+\mathbf{M}) \cdot \mathbf{W}
$$

where $\mathbf{W}$ is the six-component field, $(\mathbf{E}, c \mathbf{B}), \mathbf{S}$ is the source term, $\left(-\frac{\mathbf{J}}{\varepsilon_{0}}, \mathbf{0}\right)$, and the operators $\mathbf{P}$ and $\mathbf{M}$ are defined by

$$
\mathbf{P} \cdot \mathbf{W}=\mathbf{P} \cdot\left[\begin{array}{c}
E_{x} \\
E_{y} \\
E_{z} \\
c B_{x} \\
c B_{y} \\
c B_{z}
\end{array}\right] \equiv c\left[\begin{array}{c}
c \partial B_{z} / \partial y \\
c \partial B_{x} / \partial z \\
c \partial B_{y} / \partial x \\
\partial E_{y} / \partial z \\
\partial E_{z} / \partial x \\
\partial E_{x} / \partial y
\end{array}\right]
$$

and

$$
\mathbf{M} \cdot \mathbf{W}=\mathbf{M} \cdot\left[\begin{array}{c}
E_{x} \\
E_{y} \\
E_{z} \\
c B_{x} \\
c B_{y} \\
c B_{z}
\end{array}\right] \equiv-c\left[\begin{array}{c}
c \partial B_{y} / \partial z \\
c \partial B_{z} / \partial x \\
c \partial B_{x} / \partial y \\
\partial E_{z} / \partial y \\
\partial E_{x} / \partial z \\
\partial E_{y} / \partial x
\end{array}\right]
$$

As in Ref. [15] we use the mnemonic that $\mathbf{P}$ has a plus sign on the right, while the operator $\mathbf{M}$ has a minus sign.

The discrete representation of Eq. (7) is obtained by assuming the electric and magnetic fields are laid out as described previously. That is, the electric field values are located at the centers of cell edges (with index $(i, j, k)$ ) while the magnetic fields are located at the centers of cell faces (with index $(i, j, k)$ ). Therefore, the discrete representation of Eq. (7) is given by

$$
\mathbf{P}_{h} \cdot \mathbf{W}_{h} \equiv c\left[\begin{array}{c}
c \cdot l_{z}\left(B_{z, i, j, k}-B_{z, i, j-1, k}\right) / A_{y z} \\
c \cdot l_{x}\left(B_{x, i, j, k}-B_{x, i, j, k-1}\right) / A_{x z} \\
c \cdot l_{y}\left(B_{y, i, j, k}-B_{y, i-1, j, k}\right) / A_{x y} \\
l_{y}\left(E_{y, i, j, k+1}-E_{y, i, j, k}\right) / A_{y z} \\
l_{z}\left(E_{z, i+1, j, k}-E_{z, i, j, k}\right) / A_{x z} \\
l_{x}\left(E_{x, i, j+1, k}-E_{x, i, j, k}\right) / A_{x y}
\end{array}\right]
$$

and

$$
\mathbf{M}_{h} \cdot \mathbf{W}_{h} \equiv-c\left[\begin{array}{c}
c \cdot l_{y}\left(B_{y, i, j, k}-B_{y, i, j, k-1}\right) / A_{y z} \\
c \cdot l_{x}\left(B_{z, i, j, k}-B_{z, i-1, j, k}\right) / A_{x z} \\
c \cdot l_{x}\left(B_{x, i, j, k}-B_{x, i, j-1, k}\right) / A_{x y} \\
l_{z}\left(E_{z, i, j+1, k}-E_{z, i, j, k}\right) / A_{y z} \\
l_{x}\left(E_{x, i, j, k+1}-E_{x, i, j, k}\right) / A_{x z} \\
l_{y}\left(E_{y, i+1, j, k}-E_{y, i, j, k}\right) / A_{x y}
\end{array}\right]
$$

where $\mathbf{W}_{h}$ is the discrete representation of $\mathbf{W}$ and $\mathbf{P}_{h}$ and $\mathbf{M}_{h}$ are the finite difference versions of the continuum operators 
$\mathbf{P}$ and $\mathbf{M}$ from above. Subsequently, the discrete Maxwell's equations without sources is given by

$$
\frac{\partial \mathbf{W}_{h}}{\partial t}=\left(\mathbf{P}_{h}+\mathbf{M}_{h}\right) \cdot \mathbf{W}_{h} \text {. }
$$

In Ref. [15] the authors compared the various secondorder ADI operators that have been proposed by Zheng et al. in Ref. [2] and Lee and Fornberg in Ref. [1]. Instead of using these operators, the authors proposed an update operator that updates $\mathbf{W}_{h}$ according to

$\mathbf{W}_{h}^{n+1}=\left(\mathbf{I}+\frac{\Delta t}{2} \mathbf{M}_{h}\right) \cdot\left(\mathbf{I}-\frac{\Delta t}{2} \mathbf{P}_{h}\right)^{-1}$.

$\left[\left(\mathbf{I}+\frac{\Delta t}{2} \mathbf{P}_{h}\right) \cdot\left(\mathbf{I}-\frac{\Delta t}{2} \mathbf{M}_{h}\right) \cdot \mathbf{W}_{h}^{n}+\Delta t \mathbf{S}^{n-1 / 2}\right]$

with the property that $\nabla^{h} \cdot \mathbf{B}_{h}=0$ and $\nabla^{h} \cdot \mathbf{E}_{h}^{n+1}-\nabla^{h} \cdot \mathbf{E}_{h}^{n}=\left(\rho^{n+1}-\rho^{n}\right) / \varepsilon_{0}$ to machine precision. We focus on this form for the ADI update of Maxwell's equations but note nothing here is specific to this form.

The extension of Eq. (10) to embedded boundaries is a direct application of the Dey-Mittra boundary algorithm presented in Sec. 2.1. For the Ampere update of $\mathbf{E}$, it was discussed in Sec. 2.1 that the electric field is set to zero for edges contained entirely within the conductor; otherwise, the update step from the Yee algorithm was not changed for electric field values on edges partially in the conductor. In terms of Eq. (7), the electric field update for edges entirely in the conductor affects $\mathbf{P}_{h}$ and $\mathbf{M}_{h}$ by setting the length coefficients on the $B_{\{x, y, z\}, i, j, k}$ coefficients to be zero such that, in Eq. (10), each of the four operators becomes the identity operator, I, for those values. Thus, for all electric field values on edges in the conductor, Eq. (10) becomes $\mathbf{W}_{h}^{n+1}=\mathbf{W}_{h}^{n}$ since we have no sources in the conductor.

For the Faraday update, we have to adjust the coefficients on the $E_{\{x, y, z\}, i, j, k}$ terms in Eq. (10) according to the fraction of the corresponding edge within the domain as was done in Eq. (1). Similarly, we have to adjust the areas for the faces according to the fraction within the domain. See Fig. (2) for reference. Thus, for magnetic field values on faces that are partially in the conductor, the contribution to $\mathbf{P}_{h}$ and $\mathbf{M}_{h}$ in Eq. (7) is altered by the fractions of the lengths of each edge and the fraction of the area of each face associated with the update. Once these changes are made to $\mathbf{P}_{h}$ and $\mathbf{M}_{h}$ the ADI update proceeds accordingly. As we will see in the next section there is no effect on the stability of the method by incorporating the Dey-Mittra boundary contributions to the update step. Furthermore, the fraction of the edges and areas within the conductor has no effect on the time step due to the implicit nature of the ADI method.

\section{RESULTS}

\subsection{Background}

To establish the stability and accuracy of the ADI-FDTD method that employs the Dey-Mittra embedded boundary approximation, we consider the extraction of frequencies from the well-studied 2D Palevsky-Bekefi A6 magnetron device [9] pictured in Fig. (3). In particular, we use the frequency extraction method presented in Refs. $[10,12]$ to determine the frequencies between 1.0 and $8.0 \mathrm{GHz}$. Previous work [16] has found the frequencies between 1.0 and $8.0 \mathrm{GHz}$ to be those given in Table $\mathbf{1}$.

We consider the accuracy of the extracted frequencies as it depends on spatial resolution and temporal resolution. We apply the frequency extraction algorithm to simulations performed with the ADI-FDTD method presented and also for reference we use the EXP-FDTD method. We show for the A6 magnetron that the factor, $f_{D M}$, can be made arbitrarily small ensuring that all cells are kept in the simulation, while maintaining stability at time steps beyond the CFL condition.

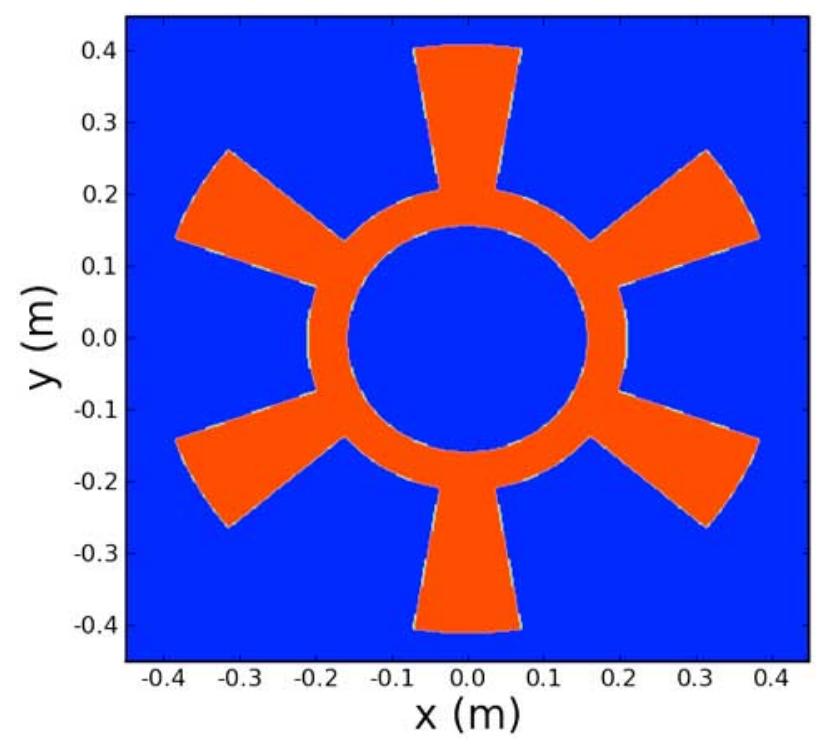

Fig. (3). The 2D Palevsky-Bekefi A6 magnetron device for which the ADI method was used for extracting frequencies with the broadlyfiltered diagonalization method of Ref. [10]. Note that the magnetron is $0.08 \mathrm{~m}$ by $0.08 \mathrm{~m}$ while the domain is $0.09 \mathrm{~m}$ by $0.09 \mathrm{~m}$.

Before presenting any results, we briefly describe the method presented in Ref. [10, 12] to give the reader a context for the simulations. The frequency extraction method has two phases. The first phase is the "ring-up" phase during which a Gaussian-modulated signal is applied to the Maxwell's equations (through a current source) such that only the frequencies between 1.0 and $8.0 \mathrm{GHz}$ are excited.

Table 1. Frequencies (in GHz) of the 2D Palevsky-Bekefi A6 Magnetron Between 1.0 and 8.0 GHz as Presented in Ref. [16]

\begin{tabular}{|c|c|c|c|c|c|c|}
\hline $\boldsymbol{f}_{\mathbf{0}}$ & $\boldsymbol{f}_{\mathbf{1}}$ & $\boldsymbol{f}_{\mathbf{2}}$ & $\boldsymbol{f}_{\mathbf{3}}$ & $\boldsymbol{f}_{\mathbf{4}}$ & $\boldsymbol{f}_{\mathbf{5}}$ & $\boldsymbol{f}_{\mathbf{6}}$ \\
\hline \hline 1.3863006 & 2.1580965 & 2.3580981 & 4.6399716 & 5.0347274 & 6.2597383 & 7.69322776 \\
\hline
\end{tabular}


The current, $\mathbf{J}(x, y, z, t)$, is given by $\mathbf{J}(x, y, z, t)=f(t) \hat{\mathbf{J}}(x, y, z)$ where

$$
f(t)= \begin{cases}2\left[\frac{\sin \left(\omega_{1}(t-T / 2)\right)}{t-T / 2}-\frac{\sin \left(\omega_{2}(t-T / 2)\right)}{t-T / 2}\right] \exp ^{-\sigma_{\omega}^{2}(t-T / 2)^{2} / 2} & 0 \leq t \leq T, \\ 0 . & \text { otherwise }\end{cases}
$$

where $\omega_{1}=2.0 e 9 \cdot \pi$ and $\omega_{2}=16.0 e 9 \cdot \pi . \hat{\mathbf{J}}(x, y, z)$ has a pattern that encourages excitation of the desired modes in the frequency range $\left[\omega_{1}, \omega_{2}\right]$. The parameter $\sigma_{\omega}$ is determined by the separation of the frequencies in $\left[\omega_{1}, \omega_{2}\right]$ from the next nearest frequency value. If $\hat{\omega}<\omega_{1}$ is the nearest frequency, then

$\sigma_{\omega}<\frac{\left|\omega_{1}-\hat{\omega}\right|}{5.68}$

and

$T>\frac{11.4}{\sigma_{\omega}}$

ensures that $\hat{\omega}$ and all other outside modes are suppressed by at least $\mathrm{O}(1 e-7)$. The second phase of the frequency extraction approach is where the fields are sampled and small scale linear algebra is performed to determine the frequencies of the modes found between 1.0 and $8.0 \mathrm{GHz}$. At this time, the corresponding mode patterns can also be constructed during the determination of frequencies. Additional details of the techniques are found in Ref. [10].

\subsection{Frequency Convergence Results}

The number of grid points considered is defined by $N x=N y=50 * k$ where $k=1,2,4,8$ yielding resolutions from $0.018 \mathrm{~m}$ to $0.00225 \mathrm{~m}$. The factor, $f_{D M}$, is chosen to be $0.1,0.3$, and 0.5 which defines the time step necessary for stability. We compare computed frequency values for the EXP-FDTD method with these values and for the ADIFDTD method. At each $f_{D M}$ value, a certain number of cutcells are discarded due to their size relative to the size of the typical cell of the domain. See Table 2 for the percentage of cells thrown away and the corresponding time step of various grid resolutions and $f_{D M}$ values.
The important case is when $f_{D M} \approx 0$ so that no cut-cells are discarded as would be required by the EXP-FDTD method. Using such a small $f_{D M}$ for the EXP-FDTD method would imply that the time step would be effectively zero. In Fig. (4) we consider two cases (last two in legend) where $f_{D M} \approx 0$. One of these uses a time step that is equivalent to setting $f_{D M} \approx 0.5(A D I-0.5)$ and the other uses a time step that is $2 \mathrm{x}$ the CFL limit $(A D I-2.0)$. In Table 2 we state the time steps generated by these methods and the corresponding percentage of cells thrown out with $f_{D M} \approx 0$. The convergence of the first four modes is plotted in Fig. (4). The last three modes exhibited similar convergence behavior. We have also included in Fig. (5a) color contour plot of the $z$ component of the magnetic field for the first four modes computed with $A D I-0.5$.

\section{DISCUSSION}

\subsection{Stability}

As is illustrated in Fig. (4), we maintain stability and can extract accurate frequencies at $2 \mathrm{x}$ the CFL limit. To further illustrate stability, we have performed simulations with the ADI-FDTD method from $1 \mathrm{x}$ to $8 \mathrm{x}$ the CFL limit. Fig. (6) shows the results of convergence after running the simulation at these time steps beyond the CFL limit. All simulations are stable and permit extraction of frequencies. In this figure, we have also included the convergence for the EXP-FDTD method at $f_{D M}=0.1$. These results are the most accurate due to the highest temporal resolution. What is clearly observed from the results in Fig. (6) is that the time step with the ADI-FDTD method can be chosen for accuracy considerations instead of stability considerations. Performing simulations with the EXP-FDTD method at $1 \mathrm{x}$ to $8 \mathrm{x}$ the CFL limit would lead to unstable calculations. Thus, the ADIFDTD method yields stable simulations well beyond the CFL limit for a geometry with a curved domain as in Fig. (3).

\subsection{Accuracy}

As has been noted previously, the focus of this work is not on the accuracy of the frequency extraction approach with the ADI-FDTD approach. The accuracy is determined by both the discretization approach and the spatial and temporal resolution. This has been studied in Ref. [17] where the authors use the EXP-FDTD method to run the

Table 2. Time Step in $p s$ for Each $N x$ and $f_{D M}$ Value (left) and the Percentage of cut-Cells Discarded from the Simulation Given the $f_{D M}$ Value (Right) Note that for CFL2 the Time Stepping is Performed via the ADI-FDTD Method so we can Include All Cells in the Simulation since we are Not Limited by the CFL Condition

\begin{tabular}{|c|c|c|c|c|}
\hline $\mathbf{N x}$ & $\mathbf{f}_{\mathbf{D M}}=\mathbf{0 . 1}$ & $\mathbf{f}_{\mathbf{D M}}=\mathbf{0 . 3}$ & $\mathbf{f}_{\mathbf{D M}}=\mathbf{0 . 5}$ & $\mathbf{f}_{\mathbf{D M}}=\mathbf{2 . 0}$ \\
\hline \hline 50 & $0.41607 / 0.0 \%$ & $1.24820 / 5.7 \%$ & $2.08033 / 11 \%$ & $6.64498 / 0.0 \%$ \\
\hline 100 & $0.20803 / 0.0 \%$ & $0.62410 / 4.0 \%$ & $1.04017 / 11 \%$ & $3.94493 / 0.0 \%$ \\
\hline 200 & $0.10402 / 0.5 \%$ & $0.31205 / 4.5 \%$ & $0.52008 / 14 \%$ & $2.08199 / 0.0 \%$ \\
\hline 400 & $0.05201 / 0.6 \%$ & $0.15602 / 4.8 \%$ & $0.26004 / 12 \%$ & $1.56174 / 0.0 \%$ \\
\hline
\end{tabular}


(a) $1.386300 \mathrm{E}+09 \mathrm{~Hz}$

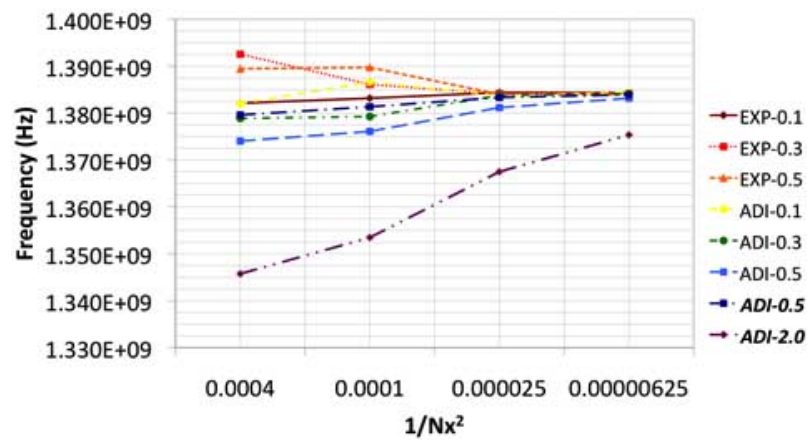

(b) $2.1580965 \mathrm{E}+09 \mathrm{~Hz}$

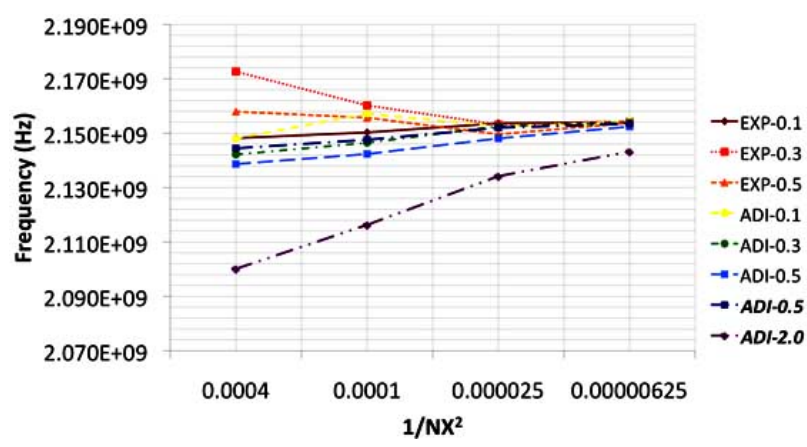

(c) $2.3580981 \mathrm{E}+09 \mathrm{~Hz}$

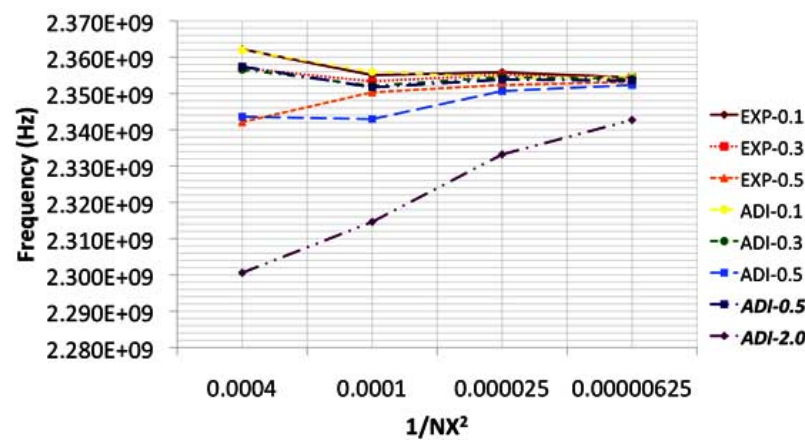

(d) $4.6399716 \mathrm{E}+09 \mathrm{~Hz}$

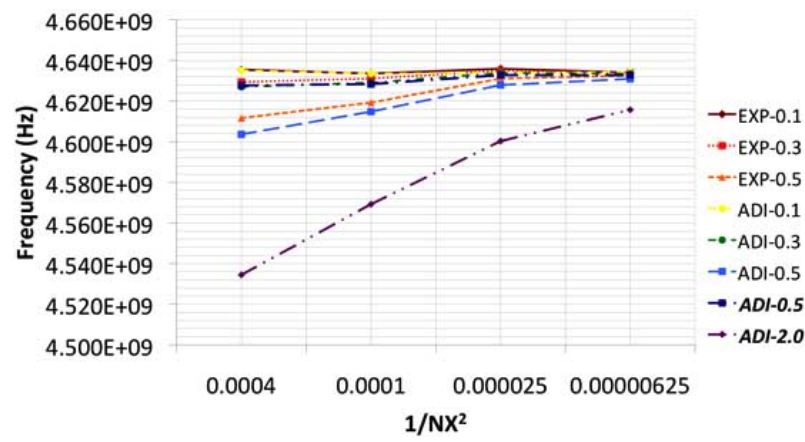

Fig. (4). Convergence of magnetron frequencies for an explicit FDTD method and an alternating direction implicit FDTD methods with Dey-Mittra cut-cells [8]. The frequency extraction approach of Werner and Cary in Ref. [10] is used to obtain the frequencies from the time domain simulations. The values $0.1,0.3$, and 0.5 refer to the $f_{D M}$ values discussed previously that are used to determine the time step and the percentage of cells kept in the simulation (see Table 2). The final two in the legend in bold have time steps determined by $f_{D M}$ but keep all of the cells in the simulation. This is only possible because of the implicit nature of the time stepping. (a) $1.386300 \mathrm{E}+09 \mathrm{~Hz}$

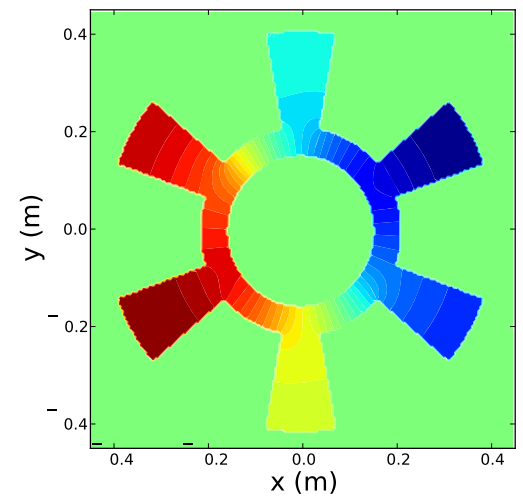

(b) $2.1580965 \mathrm{E}+09 \mathrm{~Hz}$

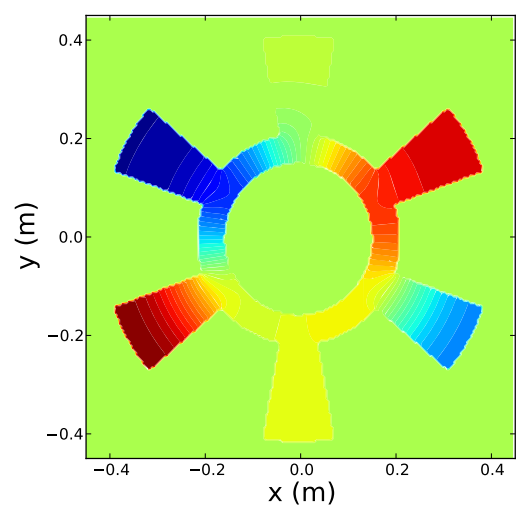

(c) $2.3580981 \mathrm{E}+09 \mathrm{~Hz}$

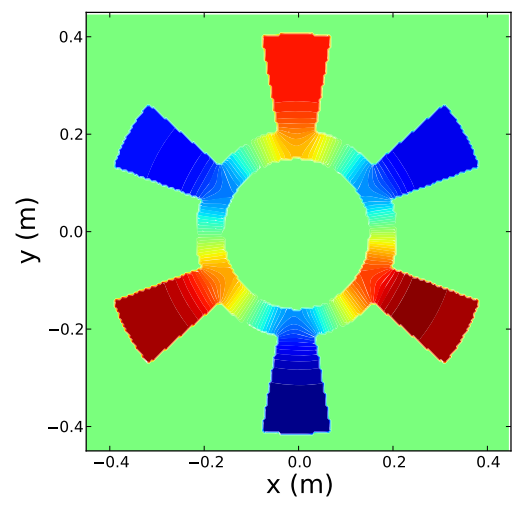

(d) $4.6399716 \mathrm{E}+09 \mathrm{~Hz}$

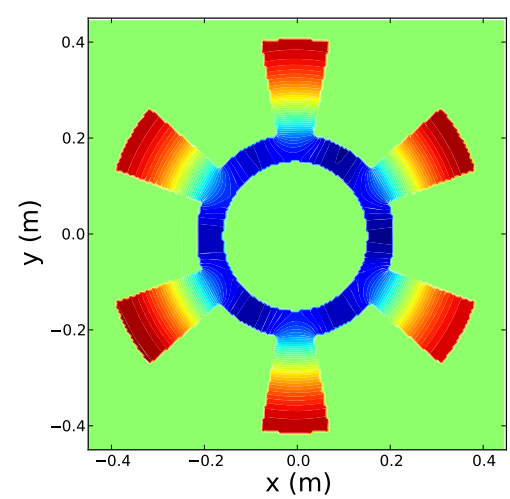

Fig. (5). Color contour plots of $B_{z}$ for the first four modes calculated using the frequency extraction method with simulations performed by the ADI-FDTD method. Results illustrate our ability to use ADI-FDTD simulations to also reconstruct spatial mode patterns. 
simulations with $f_{D M}=0.25$ and $f_{D M}=0.5$. In Fig. (4) we observe that the EXP-FDTD approach and the ADI-FDTD approach both have comparable accuracy at the same time steps. Furthermore, the example given by $A D I-0.5$ with no cells discarded shows comparable accuracy to $A D I-0.3$ with cells thrown out according to $f_{D M} \approx 0$. This tells us that we can obtain comparable accuracy with nearly twice the time step size by using ADI-FDTD and not discarding any cells. Finally, we note that with the ADI method we can go beyond the CFL time step. This example denoted as ADI-0.5 shows that the accuracy is poorer because of the lower resolution in temporal space. Furthermore, with Fig. (6), we see that reducing the time step size improves the accuracy of the computations.

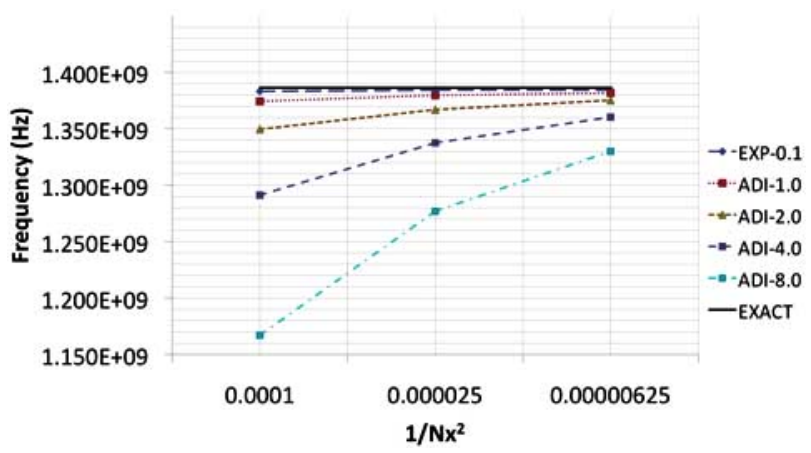

Fig. (6). Convergence of the ADI-FDTD method at various temporal resolutions, from $1 \mathrm{x}$ the CFL limit to $8 \mathrm{x}$ the CFL limit. Also included on this convergence plot is the convergence for EXPFDTD at $f_{D M}=0.1$ and the exact value, as obtained from [9], plotted as a flat line at $1.3836 \mathrm{GHz}$. The ADI-FDTD method remains stable at all time steps beyond the CFL limit and only exhibits lower accuracy than at time steps below the CFL limit.

\section{CONCLUSION}

We have presented an implementation of the ADI-FDTD method combined with the Dey-Mittra embedded boundary method. This approach can model the curved domains associated with complex accelerator structures and at time step sizes beyond the CFL limit. It depends on simple, onedimensional, tridiagonal solves instead of the large system solves associated with implicit methods like the CrankNicholson method. The one-dimensional solves can be efficiently completed using the Thomas algorithm [18].

In three-dimensions, the method can be directly applied without any changes. Clearly, for large three-dimensional problems, the method must be extended to a large scale parallel computing platform to handle the larger number of unknowns. An efficient implementation thus depends on the efficient solution of a number of tridiagonal systems at each time step. We leave this to future work.

\section{REFERENCES}

[1] Lee J, Fornberg B. Some unconditionally stable time stepping methods for the 3D Maxwell's equations. J Comput Appl Math 2004; 166(2): 497-523.

[2] Zheng F, Chen Z, Zhang J. Toward the development of a threedimensional unconditionally stable finite-difference time-domain method. IEEE Trans Microw Theory Tech 2000; 48(9): 1550-8.

[3] Namiki T. 3-D ADI-FDTD method-unconditionally stable timedomain algorithm for solving full vector Maxwell's equations. IEEE Trans Microw Theory Tech 2000; 48(10): 1743-8.

[4] Zhao AP. Two special notes on the implementation of the unconditionally stable ADI-FDTD method. Microw Opt Technol Lett 2002; 33(4)

[5] Zivanovic SS, Yee KS, Mei KK. A subgridding method for the time-domain Vnite-diVerence method to solve Maxwell's equations. IEEE Trans Microw Theory Tech 1991; 39(3): 471-9.

[6] Ahmed I, Chen ZD. A hybrid ADI-FDTD subgridding scheme for eVंcient electromagnetic computation. Int J Numer Modell Electron Netw Devices Fields 2004; 17(3).

[7] Yee KS. Numerical solution of initial boundary value problems involving Maxwell's equations in isotropic media. IEEE Trans Antennas Propag 1966; 14: 302.

[8] Dey S, Mittra R. A locally conformal finite-difference time-domain (FDTD) algorithm for modeling three-dimensional perfectly conducting objects. IEEE Microw Guided Wave Lett 1997; 7(9): 273-5.

[9] Palevsky A, Bekefi G. Microwave emission from pulsed, relativistic e-beam diodes. II. The multiresonator magnetron. Phys Fluids 1979; 22: 986.

[10] Werner GR, Cary JR. Extracting degenerate modes and frequencies from time-domain simulations with filter-diagonalization. J Comput Phys 2008; 227(10): 5200-214.

[11] Nieter C, Cary JR. VORPAL: A versatile plasma simulation code. J Comput Phys 2004; 196(2): 448-73.

[12] Austin TM, Cary JR, Werner GR, Bellantoni L. Validation of broadly filtered diagonalization method for extracting frequencies and modes from high-performance computations. J Phys Conf Ser 2009; 180: 012003.

[13] Nieter C, Cary JR, Smithe D, Stoltz PH, Werner GR. Simulations of electron eVects in superconducting cavities with the VORPAL code. In: Proc EPAC 2006; pp. 2269-71.

[14] Nieter C, Cary JR, Werner GR, Smithe DN, Stoltz PH. Application of Dey-Mittra conformal boundary algorithm to 3D electromagnetic modeling. J Comput Phys 2009; 228(21): 7902-16.

[15] Smithe DN, Cary JR, Carlsson JA. Divergence preservation in the ADI algorithms for electromagnetics. J Comput Phys 2009; 228(19): 7289-99.

[16] Davidson RC, Chan HW, Chen C, Lund S. Equilibrium and stability properties of intense non-neutral electron flow. Rev Mod Phys 1991; 63(2): 341-74.

[17] Lin MC, Nieter C, Stoltz PH, Smithe DN. Accurately and eVंciently studying the RF structures using a conformal VinitediV̈erence time-domain particle-in-cell method; Submitted to Open Plasma Phys J Special Issue on Recent Advances in Finite Difference Time Domain Electromagnetic Simulations.

[18] Thomas LH. Elliptic problems in linear diVerence equations over a network. Watson Sci Comput Lab Rept, New York: Columbia University 1949. 